\title{
IVESR Rumor Spreading Model in Homogeneous Network with Hesitating and Forgetting Mechanisms
}

\author{
Md. Nahid Hasan, Saiful Islam*, Chandra Nath Podder \\ Department of Mathematics, University of Dhaka, Dhaka 1000, Bangladesh.
}

\begin{abstract}
How to cite this paper: Md. Nahid Hasan, Saiful Islam, Chandra Nath Podder. (2021) IVESR Rumor Spreading Model in Homogeneous Network with Hesitating and Forgetting Mechanisms. Journal of Applied Mathematics and Computation, 5(2), 105-118.

DOI: 10.26855/jamc.2021.06.005
\end{abstract}

Received: April 5, 2021

Accepted: April 30, 2021

Published: May 21, 2021

*Corresponding author: Saiful Islam, Department of Mathematics, University of Dhaka, Dhaka 1000, Bangladesh.

Email: sislam@du.ac.bd

\begin{abstract}
In this paper, we study a new IVESR rumor spreading model with hesitating and forgetting mechanisms in homogeneous network. The rumor free and rumor prevailing equilibriums, and the basic reproduction number $\mathfrak{R}_{0}$ are calculated from the mean-field equations of the model. The local and global stability of rumor free equilibrium are proved by using Lyapunov function and LaSalle invariance principle, and the existence of rumor prevailing equilibrium is shown. In numerical simulations, it is found that the vaccination, prohibiting people to spread the rumor, can lessen the propagation of rumor in the network. We also show that the fuzziness of the rumor has a great impact on the size of spreader and the forgetting factor has a great effect on the rumor prevailing duration. Furthermore, we analyze the sensitivity of different parameters on $\mathfrak{R}_{0}$ and discussed how they affect the spreading and controlling of the rumor.
\end{abstract}

\section{Keywords}

Rumor Spreading, Epidemic Model, Vaccination, Basic Reproduction Number, Sensitivity Analysis

\section{Motivation}

Rumor is usually defined as the unconfirmed elaboration or annotation of the public interesting things, events or issues that spread through various channels, in itself neither true nor false [1-4]. Rumor is a form of social communication and can shape the public opinion and affects the beliefs of individuals, which can lead to the changes of individual's attitude towards economic, political, and social aspects [5, 6]. Because of the increased presence of online social networks, rumors are no longer spread by word of mouth over a small area but are spread amongst strangers in different regions and different countries. Rumors are part of our everyday life. As an important part of people's lives, rumors are being used as a special weapon of public opinion and can pose a tremendous impact on social life [7], as well as on financial markets and their spreading has the ability to shape public opinion [8] and lead to social panic and instability afterward [9, 10]. Because of the similar propagation mechanism between epidemic spreading and rumor propagation, epidemic models have been widely used in revealing the rumor propagation dynamics of online social networks.

The study of rumor-spreading models began in the 1940s. Based on the SIR epidemic model, Daley and Kendall $[7,11]$ proposed the basic DK model, the beginning of rumor spreading modeling, in the 1960s. In their model, the population is subdivided into three groups: those who are unaware of the rumor (ignorants), those who spread the rumor (spreaders), and those who are aware of the rumor but choose not to spread it (stiflers). After that, Maki [12] modified the DK model and developed the MK model, in which rumors propagate through direct contact between spreaders and others. Afterwards, Nekovee et al. [13] and Isham et al. [14] built a new model by combining the MK model with the SIR epidemic 
model on complex networks. Gu et al. $[15,16]$ and Zhao et al. $[17,18]$ refined the rumor spreading model by incorporating the effects of remembering mechanism in complex networks. Wan et al. [19] developed a SEIR rumor spreading model with demographics on scale-free networks. Liu et al. [20] developed a SEIR rumor spreading model with hesitating mechanism. Zhou et al. [21] worked on how to detect source of rumor in networks based on the SEIR model.

So, all the above study is about how rumor spreads, spreading mechanisms, spreading behavior etc. In this article, we formulate a novel deterministic model to analyze how vaccination that is the prohibition to spread the rumor can control the propagation. Suppose in a country, a rumor has started spreading and is creating an unstable situation in the society. Then the government announces that this information is wrong and all the government officials are prohibited not to believe and spread this information. Then, the government officials will act like the vaccinated individuals. Also, there are electronic media and other volunteers who are working to make people conscious about the rumor. Then, in this situation what will be the spreading behavior of the rumor? Moreover, it is natural that a government party activist will not believe the rumor against the government as well as the opposition party activists will also distrust the rumor against the opposition party. So, they act like vaccinated in their respective situations. In this paper, we analyze the mechanisms and behavior of rumor spreading in those situations with a more realistic mathematical model including hesitating and forgetting mechanisms. Considering all of these realistic cases of rumor spreading, we formulate a new IVESR (Ignorant, Vaccinated, Exposed, Spreader, Stifler) model and analyze qualitatively and numerically.

\section{Model Formulation}

Firstly, we consider the whole population is located in a relevant social network. While propagating the rumor the whole population is divided into five distinct classes of Ignorant (I), Vaccinated (V), Exposed (E), Spreader (S), Stifler (R). Ignorants are those who have never contacted with the rumor and are susceptible of being informed of the rumor. Vaccinated are those who are being prohibited to spread the rumor or being made conscious about the rumor. Exposed are those individuals who have been informed of the rumor but they are in hesitating state whether to spread the rumor or not and have not spread the rumor yet. The individuals who are actively spreading the rumor are Spreaders, and Stiflers are those individuals who have known the rumor but will not spread it anymore. During the time of rumor spreading in an area there are people who enter into the area and people who leave. Also, there are people who actively use the social networks or create new accounts and there are people who are inactive in social networks or deactivated their account. That's why we assume the population is open. So, the dynamics include entering and leaving, and in our model, we consider the new entering population are all the Ignorants.

In the rumor spreading process, the spreading procedure among these five compartments is governed by the following rules: we consider the homogeneous network and $\bar{k}$ is the average degree of the network. When a spreader contacts an ignorant then the ignorant transfer to exposed compartment with a probability $\lambda_{1}$, that is, with the contacts of the spreaders the ignorants come to know about the rumor and start hesitating whether they spread it or not. After contacting with spreaders a few ignorant individuals do not trust the rumor and they transfer to stifler compartment at a rate $\lambda_{3}$. Besides these, due to the official announcement of prohibition of spreading of the rumor and due to the volunteers' campaign, some ignorant individuals shift to vaccinated compartment with a rate $\lambda_{2}$. Later from the vaccinated compartment some vaccinated individuals while contacting with the spreaders turn into spreader and shift to spreader compartment with a probability $\sigma$. We consider $h(0 \leq h \leq 1)$ as the attractiveness of rumor itself on the social networks, and the corresponding repellent of rumor is $(1-h)$. From the exposed compartment the hesitating individuals transfer to spreader at a probability $\tau h$ and transfer to stifler at probability $\tau(1-h)$. Now, the fuzziness of rumor is $m(0 \leq m \leq 1)$ and the corresponding clarity of rumor is $(1-m)$ and the forgetting rate of the rumor is $\alpha$. Due to the fuzziness of the rumor, the spreaders forget the rumor and transfer to ignorant individuals at a rate $\alpha m$ and due to losing interest at the rumor, spreaders transfer to stifler at a rate $\alpha(1-m)$. Also, the entering rate of ignorant individuals is $\beta$ and leaving rate of individuals from any compartment is $\mu$. The IVESR rumor spreading procedure is shown in Figure 1.

Considering all the above rules, we obtain the following mean field equations. The meaning of the parameters in the model are described in Table 1.

$$
\begin{gathered}
\frac{d I(t)}{d t}=\beta+m \alpha S(t)-\bar{k} \lambda_{3} S(t) I(t)-\bar{k} \lambda_{1} I(t) S(t)-\lambda_{2} I(t)-\mu I(t) \\
\frac{d V(t)}{d t}=\lambda_{2} I(t)-\bar{k} \sigma S(t) V(t)-\mu V(t) \\
\frac{d E(t)}{d t}=\bar{k} \lambda_{1} I(t) S(t)-\tau E(t)-\mu E(t) \\
\frac{d S(t)}{d t}=\tau h E(t)+\bar{k} \sigma S(t) V(t)-\alpha S(t)-\mu S(t)
\end{gathered}
$$




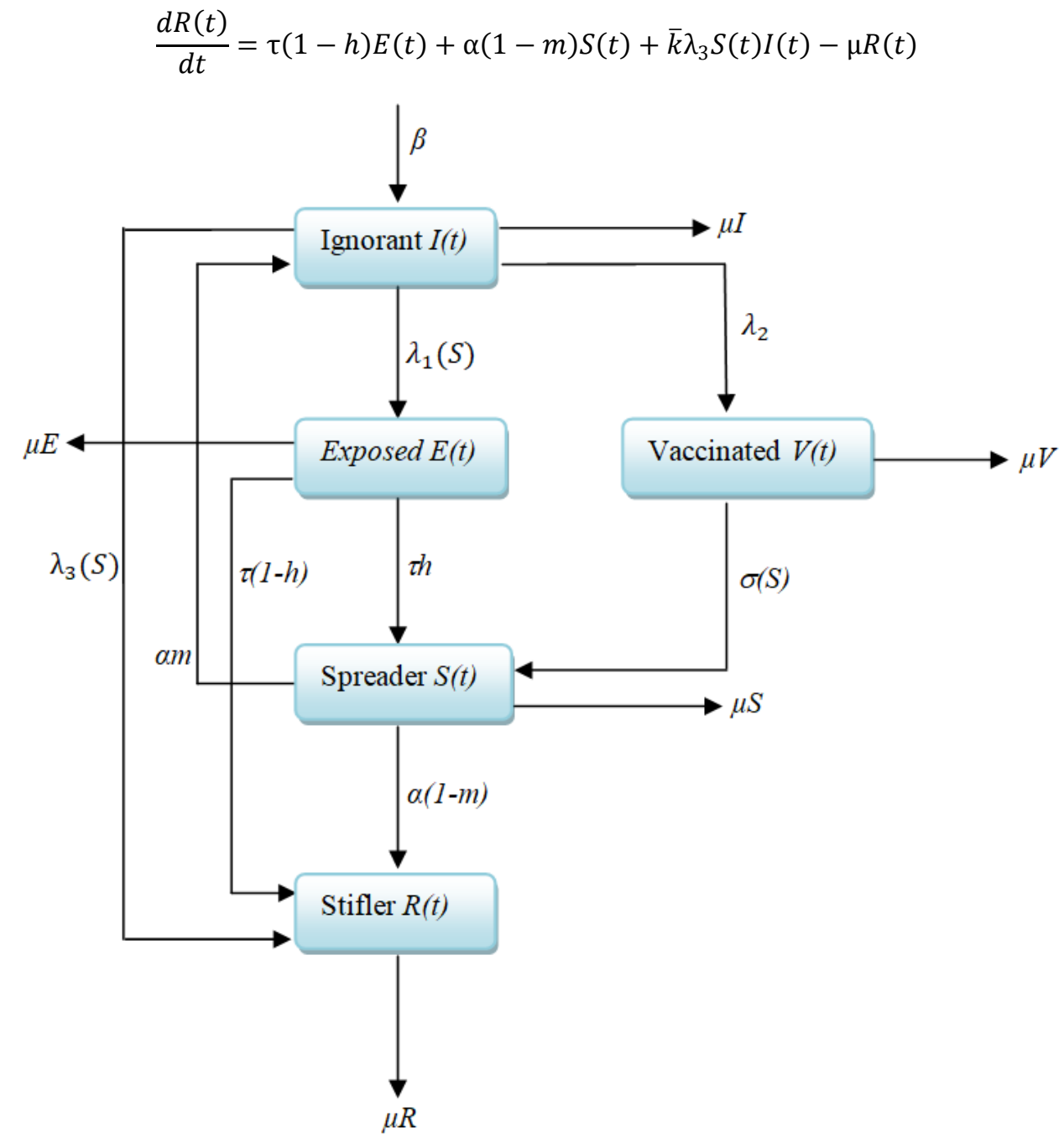

Figure 1. The schematic diagram of the IVESR model for the rumor spreading process.

Table 1. The description of the parameters used in the model (1)

\begin{tabular}{ccc}
\hline $\boldsymbol{\beta}$ & Explaination \\
$\boldsymbol{\mu}$ & Entering rate of ignorants \\
$\lambda_{\mathbf{1}}$ & Leaving rate of the population \\
$\lambda_{2}$ & Rate of people being made conscious by the government and volunteers \\
$\lambda_{3}$ & Rate at which the ignorant distrust the rumor \\
$\boldsymbol{\sigma}$ & Transfer rate from vaccinated to spreader due to contacts \\
$\boldsymbol{\tau}$ & Transmission probability from exposed to spreader \\
$\boldsymbol{\alpha}$ & Attractiveness of the rumor, where $0 \leq h \leq 1$ \\
$\boldsymbol{m}$ & Forgetting rate of the rumor \\
\hline
\end{tabular}

The population density of Ignorants, Vaccinated, Exposed, Spreaders, and Stiflers at any time $t$ are denoted by $I(t)$, $V(t), E(t), S(t)$ and $R(t)$ respectively, and the total number of individuals is $N(t)$ and we have $I+V+E+S+$ $R=N$. 


\section{Analysis of the Model}

\subsection{Properties of the Model}

Here we prove some basic qualitative properties of solutions to the model (1), such as positivity and boundedness of the solutions. These properties can be shown by the following lemma:

Lemma 3.1.1: The region, $\Omega=\left\{(I, V, E, S, R): I+V+E+S+R \leq \frac{\beta}{\mu}\right\} \subset R_{+}^{5}$ is positively invariant and attracting for the basic model (1).

Proof: The rate of change of the individuals is obtained by adding the five equations of the model (1) as follows

$$
\frac{d N(t)}{d t}=\beta-\mu N(t)
$$

Thus, we see that $\frac{d N(t)}{d t}<0$ if $N(t)>\frac{\beta}{\mu}$. Also, by using a standard comparison theorem [22], it can be shown that $N(t)=\frac{\beta}{\mu}+\left(N(0)-\frac{\beta}{\mu}\right) e^{-\mu t}$. In particular, $N(t)<\frac{\beta}{\mu}$ if $N(0)<\frac{\beta}{\mu}$. Thus, $\Omega$ is positively invariant. Further, $N(t)>\frac{\beta}{\mu}$, then either the solution enters $\Omega$ in finite time, or $N(t)$ approaches $\frac{\beta}{\mu}$, and the variables $V(t), E(t), S(t)$ and $R(t)$ approach zero. Hence, $\Omega$ is attracting. Thus, the model (1) is well-posed in $\Omega$ epidemiologically and mathematically [23] and it is sufficient to study the dynamics of the model (1) in $\Omega$.

\subsection{Rumor Free Equilibrium (RFE)}

Rumor-free equilibrium $\left(I_{0}, V_{0}, E_{0}, S_{0}, R_{0}\right)$ can be found by solving the equations, obtained from model (1) by setting $\frac{d I(t)}{d t}=0, \frac{d V(t)}{d t}=0, \frac{d E(t)}{d t}=0, \frac{d S(t)}{d t}=0$ and $\frac{d R(t)}{d t}=0$.

It is noted that for the rumor-free equilibrium, we consider the count of compartment of spreader individuals $S_{0}=0$. Then, we find $I_{0}=\frac{\beta}{\lambda_{2}+\mu}, V_{0}=\frac{\beta \lambda_{2}}{\mu\left(\lambda_{2}+\mu\right)}, E_{0}=0, S_{0}=0, R_{0}=0$. So, the rumor-free equilibrium is

$$
\bar{E}_{0}=\left(\frac{\beta}{\lambda_{2}+\mu}, \frac{\beta \lambda_{2}}{\mu\left(\lambda_{2}+\mu\right)}, 0,0,0\right)
$$

\subsection{Basic Reproduction Number $\left(\mathfrak{R}_{0}\right)$}

Now, we calculate the basic reproduction number of the model (1) according to [24]. Consider the compartments which are related to spreader to obtain the following subsystem.

$$
\begin{gathered}
\frac{d E(t)}{d t}=\bar{k} \lambda_{1} I(t) S(t)-\tau E(t)-\mu E(t) \\
\frac{d S(t)}{d t}=\tau h E(t)+\bar{k} \sigma S(t) V(t)-\alpha S(t)-\mu S(t)
\end{gathered}
$$

From the subsystem (3), we find the following transmission matrix $F$ (associated with new infection terms) and transition matrix $V$ (considering transferred terms) at rumor-free equilibrium $\overline{\mathrm{E}}_{0}$

$$
\begin{aligned}
& F=\left[\begin{array}{cc}
0 & \frac{\bar{k} \lambda_{1} \beta}{\lambda_{2}+\mu} \\
0 & \frac{\bar{k} \sigma \lambda_{2} \beta}{\mu\left(\lambda_{2}+\mu\right)}
\end{array}\right] \\
& V=\left[\begin{array}{cc}
\tau+\mu & 0 \\
-\tau h & \alpha+\mu
\end{array}\right]
\end{aligned}
$$

The basic reproduction number [24] is the spectral radius of the matrix $F V^{-1}$, that is, $\mathfrak{R}_{0}=\rho\left(F V^{-1}\right)$.

Therefore, it follows that the basic reproduction number of the model is

$$
\Re_{0}=\frac{\bar{k} \beta \lambda_{2} \sigma}{\mu(\alpha+\mu)\left(\lambda_{2}+\mu\right)}+\frac{\bar{k} \beta h \lambda_{1} \tau}{(\mu+\tau)(\alpha+\mu)\left(\lambda_{2}+\mu\right)}
$$

\subsection{Local Stability of $\operatorname{RFE}\left(\overline{\mathbf{E}}_{\mathbf{0}}\right)$}

For local stability of the model at rumor-free equilibrium $\left(\bar{E}_{0}\right)$, the Jacobian matrix of the model (1) at $\bar{E}_{0}$ is 


$$
J\left(\bar{E}_{0}\right)=\left[\begin{array}{ccccc}
\left(-\lambda_{2}-\mu\right) & 0 & 0 & \left(\frac{-\beta \bar{k}}{\lambda_{2}+\mu}\left(\lambda_{1}+\lambda_{3}\right)+\alpha m\right) & 0 \\
\lambda_{2} & -\mu & 0 & \frac{-\bar{k} \sigma \lambda_{2} \beta}{\mu\left(\lambda_{2}+\mu\right)} & 0 \\
0 & 0 & -(\tau+\mu) & \frac{\bar{k} \lambda_{1} \beta}{\lambda_{2}+\mu} & 0 \\
0 & 0 & \tau h & \left(\frac{\bar{k} \sigma \lambda_{2} \beta}{\mu\left(\lambda_{2}+\mu\right)}-\alpha-\mu\right) & 0 \\
0 & 0 & \tau(1-h) & \left(\frac{\bar{k} \lambda_{3} \beta}{\lambda_{2}+\mu}+\alpha(1-m)\right) & -\mu
\end{array}\right]
$$

Expanding the determinant of the characteristic equation $|J-\lambda I|=0$ by the first column, then second column and then by the last column, we obtain three of the eigenvalues of $J:-\left(\lambda_{2}-\mu\right),-\mu$ and $-\mu$. The remaining two eigenvalues are the eigenvalues of the $2 \times 2$ matrix:

$$
J_{1}\left(\bar{E}_{0}\right)=\left[\begin{array}{cc}
-(\tau+\mu) & \frac{\bar{k} \lambda_{1} \beta}{\lambda_{2}+\mu} \\
\tau h & \left(\frac{\bar{k} \sigma \lambda_{2} \beta}{\mu\left(\lambda_{2}+\mu\right)}-\alpha-\mu\right)
\end{array}\right]
$$

Then, we obtain the following characteristic polynomial of the matrix $J_{1}$

$$
\lambda^{2}+a_{1} \lambda+a_{2}=0
$$

where the coefficients are the following expressions of the parameters:

$$
\begin{gathered}
a_{1}=\alpha+\tau+2 \mu-\frac{\bar{k} \sigma \lambda_{2} \beta}{\mu\left(\lambda_{2}+\mu\right)} \\
a_{2}=\alpha \mu+\mu^{2}+\alpha \tau+\mu \tau-(\mu+\tau) \frac{\bar{k} \sigma \lambda_{2} \beta}{\mu\left(\lambda_{2}+\mu\right)}-\frac{\bar{k} \tau h \lambda_{1} \beta}{\lambda_{2}+\mu}
\end{gathered}
$$

Now, according to Routh-Hurwitz criteria the rumor-free equilibrium is locally asymptotically stable if

$$
\begin{aligned}
\Rightarrow \frac{\bar{k} \beta \lambda_{2} \sigma}{\mu(\alpha+\mu)\left(\lambda_{2}+\mu\right)}+\frac{a_{2}>0}{(\mu+\tau)(\alpha+\mu)\left(\lambda_{2}+\mu\right)}<1 \\
\Rightarrow \Re_{0}<1
\end{aligned}
$$

So, we have if $\mathfrak{R}_{0}<1$ then the $\boldsymbol{R F} \boldsymbol{E}$ is locally asymptotically stable, that is the rumor will not persist in the network.

\subsection{Global Stability of $\operatorname{RFE}\left(\overline{\mathbf{E}}_{\mathbf{0}}\right)$}

Before to prove the global stability of the $\boldsymbol{R F E}$, we consider the region,

$$
\Omega^{*}=\left\{(I, V, E, S, R) \in \Omega: I \leq I_{0}, V \leq \mathrm{V}_{0}\right\}
$$

and prove the following lemma:

Lemma 3.5.1: The region $\Omega^{*}$ is positively invariant and attracting for the model (1).

Proof: From the first equation of the model (1), where, $I_{0}=\frac{\beta}{\lambda_{2}+\mu}$, it follows that

$$
\begin{gathered}
\frac{d I(t)}{d t}=\beta-\bar{k} \lambda_{3} S(t) I(t)-\bar{k} \lambda_{1} I(t) S(t)-\lambda_{2} I(t)-\mu I(t) \leq \beta-\lambda_{2} I(t)-\mu I(t) \\
=\left(\lambda_{2}+\mu\right)\left(\frac{\beta}{\lambda_{2}+\mu}-I(t)\right)=\left(\lambda_{2}+\mu\right)\left(I_{0}-I(t)\right)
\end{gathered}
$$

Hence, we have

$$
I(t) \leq I_{0}-\left(I_{0}-I(0)\right) e^{-\left(\lambda_{2}+\mu\right) t}
$$


Thus, if $N(t) \leq \frac{\beta}{\mu}$ and so $I(0) \leq \frac{\beta}{\mu}$, therefore it follows that either $I(t) \rightarrow I_{0}$ as $t \rightarrow \infty$, or after finite time $I(t) \leq I_{0}$, since $\frac{d I}{d t}<0$ for $I(t)>I_{0}$. Finally, from the second equation of the model (1), where, $V_{0}=\frac{\beta \lambda_{2}}{\mu\left(\lambda_{2}+\mu\right)}$, it follows that

$$
\begin{gathered}
\frac{d V(t)}{d t}=\lambda_{2} I(t)-\bar{k} \sigma S(t) V(t)-\mu V(t) \leq \lambda_{2}\left(\frac{\beta}{\lambda_{2}+\mu}\right)-\mu V(t) \\
=\mu\left(\frac{\beta \lambda_{2}}{\mu\left(\lambda_{2}+\mu\right)}-V(t)\right)=\mu\left(V_{0}-V(t)\right)
\end{gathered}
$$

Hence, we have

$$
V(t) \leq V_{0}-\left(V_{0}-V(0)\right) e^{-\mu t}
$$

Therefore, if $N(t) \leq \frac{\beta}{\mu}$ and $V(0) \leq \frac{\beta}{\mu}$, then either $V(t) \rightarrow V_{0}$ as $t \rightarrow \infty$, or after finite time $V(t) \leq V_{0}$, since $\frac{d V}{d t}<0$ for $V(t)>V_{0}$.

Now we claim the following:

Theorem 3.5.2: The $\boldsymbol{R F E}, \bar{E}_{0}$, of the model (1) is globally asymptotically stable in the region $\Omega^{*}$ if $\Re_{0}<1$.

Proof: We prove the theorem by using Lyapunov function and LaSalle Invariance Principle [25, 26]. Consider the following Lyapunov function

where, $f_{1}=\frac{\tau h}{\tau+\mu}$ and $f_{2}=1$.

$$
\mathcal{F}=f_{1} E+f_{2} S
$$

Now, the time derivative of the Lyapunov function is given by

$$
\begin{gathered}
\dot{\mathcal{F}}=f_{1} \dot{E}+f_{2} \dot{S}=\frac{\tau h}{\tau+\mu}\left(\bar{k} \lambda_{1} I(t) S(t)-\tau E(t)-\mu E(t)\right)+(\tau h E(t)+\bar{k} \sigma S(t) V(t)-\alpha S(t)-\mu S(t)) \\
=E\left(\frac{\tau h(\tau+\mu)}{\tau+\mu}\right)+S(\alpha+\mu)\left[\frac{\bar{k}\left(\tau h \lambda_{1} I+V \sigma \tau+V \sigma \mu\right)}{(\alpha+\mu)(\tau+\mu)}-1\right] \\
\leq \frac{\beta}{\mu}(\alpha+\mu)\left[\frac{\bar{k}\left(\tau h \lambda_{1} \frac{\beta}{\lambda_{2}+\mu}+\sigma \tau \frac{\beta \lambda_{2}}{\mu\left(\lambda_{2}+\mu\right)}+\sigma \mu \frac{\beta \lambda_{2}}{\mu\left(\lambda_{2}+\mu\right)}\right)}{(\alpha+\mu)(\tau+\mu)}-1\right]=\frac{\beta}{\mu}(\alpha+\mu)\left(\Re_{0}-1\right)
\end{gathered}
$$

This gives, $\dot{\mathcal{F}}<0$ if $\mathfrak{R}_{0}<1$. Therefore, it implies that the rumor-free equilibrium $\bar{E}_{0}=\left(\frac{\beta}{\lambda_{2}+\mu}, \frac{\beta \lambda_{2}}{\mu\left(\lambda_{2}+\mu\right)}, 0,0,0\right)$ is globally asymptotically stable when $\Re_{0}<1$. So, the rumor will not spread out in the network irrespective of the initial size of the nodes whenever $\mathfrak{R}_{0}<1$.

\subsection{Rumor Prevailing Equilibrium (RPE)}

We assume that the rumor-prevailing equilibrium is $\left(I^{*}, V^{*}, E^{*}, S^{*}, R^{*}\right)$. Then, we write (1) as

$$
\begin{gathered}
\beta+m \alpha S^{*}(t)-\bar{k} \lambda_{3} S^{*}(t) I^{*}(t)-\bar{k} \lambda_{1} I^{*}(t) S^{*}(t)-\lambda_{2} I^{*}(t)-\mu I^{*}(t)=0 \\
\lambda_{2} I^{*}(t)-\bar{k} \sigma S^{*}(t) V^{*}(t)-\mu V^{*}(t)=0 \\
\bar{k} \lambda_{1} I^{*}(t) S^{*}(t)-\tau E^{*}(t)-\mu E^{*}(t)=0 \\
\tau h E^{*}(t)+\bar{k} \sigma S^{*}(t) V^{*}(t)-\alpha S^{*}(t)-\mu S^{*}(t)=0 \\
\tau(1-h) E^{*}(t)+\alpha(1-m) S^{*}(t)+\bar{k} \lambda_{3} S^{*}(t) I^{*}(t)-\mu R^{*}(t)=0
\end{gathered}
$$

Here, the spreader individuals $S^{*} \neq 0$. Then, we find the count of ignorant individuals, vaccinated individuals, exposed individuals and stiflers in the following forms

$$
\begin{gathered}
I^{*}=\frac{\beta+m \alpha S^{*}}{\bar{k} \lambda_{3} S^{*}+\bar{k} \lambda_{1} S^{*}+\lambda_{2}+\mu} \\
V^{*}=\frac{\left(m S^{*} \alpha+\beta\right) \lambda_{2}}{\left(\bar{k} S^{*} \lambda_{1}+\lambda_{2}+\bar{k} S^{*} \lambda_{3}+\mu\right)\left(\mu+\bar{k} S^{*} \sigma\right)}
\end{gathered}
$$




$$
\begin{gathered}
E^{*}=\frac{\bar{k} S^{*}\left(m S^{*} \alpha+\beta\right) \lambda_{1}}{\left(\bar{k} S^{*} \lambda_{1}+\lambda_{2}+\bar{k} S^{*} \lambda_{3}+\mu\right)(\mu+\tau)} \\
R^{*}=\frac{S^{*}}{\mu}\left(\alpha(1-m)+\frac{\bar{k}\left(m S^{*} \alpha+\beta\right) \lambda_{3}}{\lambda_{2}+\bar{k} S^{*}\left(\lambda_{1}+\lambda_{3}\right)+\mu}+\frac{\bar{k} \lambda_{1} \tau(1-h)\left(m S^{*} \alpha+\beta\right)}{\left(\lambda_{2}+\bar{k} S^{*}\left(\lambda_{1}+\lambda_{3}\right)+\mu\right)(\mu+\tau)}\right)
\end{gathered}
$$

We get the count of spreader individuals by solving the following equation

$$
S^{*}=\frac{h \bar{k} S^{*}\left(m S^{*} \alpha+\beta\right) \lambda_{1} \tau}{\left(\bar{k} S^{*} \lambda_{1}+\lambda_{2}+\bar{k} S^{*} \lambda_{3}+\mu\right)\left(\alpha+\mu-\frac{\bar{k}\left(m S^{*} \alpha+\beta\right) \lambda_{2} \sigma}{\left(\bar{k} S^{*} \lambda_{1}+\lambda_{2}+\bar{k} S^{*} \lambda_{3}+\mu\right)\left(\mu+\bar{k} S^{*} \sigma\right)}\right)(\mu+\tau)}
$$

After simplifying the above equation, we get the following

As $S^{*} \neq 0$, we must have

$$
S^{*}\left(c_{1} S^{* 2}+c_{2} S^{*}+c_{3}\right)=0
$$

where,

$$
c_{1} S^{* 2}+c_{2} S^{*}+c_{3}=0
$$

$$
\begin{gathered}
c_{1}=\bar{k}^{2} \sigma\left(\alpha \lambda_{1} \mu+\alpha \lambda_{3} \mu+\lambda_{3} \mu^{2}+\lambda_{1} \mu^{2}+\alpha \lambda_{3} \tau+\lambda_{1} \mu+\lambda_{3} \mu \tau+\alpha \lambda_{1} \tau(1-h m)\right) \\
c_{2}=\bar{k} \alpha \lambda_{1} \mu^{2}+\bar{k} \alpha \lambda_{3} \mu^{2}+\bar{k} \lambda_{1} \mu^{3}+\bar{k} \lambda_{3} \mu^{3}+\bar{k} \alpha \lambda_{2} \mu \sigma-\bar{k} m \alpha \lambda_{2} \mu \sigma+\bar{k} \alpha \mu^{2} \sigma+\bar{k} \lambda_{2} \mu^{2} \sigma+\bar{k} \mu^{3} \sigma+\bar{k} \alpha \lambda_{1} \mu \tau+\bar{k} \alpha \lambda_{3} \mu \tau \\
+\bar{k} \lambda_{1} \mu^{2} \tau+\bar{k} \lambda_{3} \mu^{2} \tau+\bar{k} \alpha \lambda_{2} \sigma \tau-\bar{k} m \alpha \lambda_{2} \sigma \tau+\bar{k} \alpha \mu \sigma \tau+\bar{k} \lambda_{2} \mu \sigma \tau+\bar{k} \mu^{2} \sigma \tau-h m \bar{k} \alpha \lambda_{1} \mu \tau-h \bar{k}^{2} \beta \lambda_{1} \sigma \tau \\
c_{3}=\mu(\alpha+\mu)\left(\lambda_{2}+\mu\right)(\mu+\tau)-\bar{k} \beta\left(\lambda_{2} \mu \sigma+h \lambda_{1} \mu \tau+\lambda_{2} \sigma \tau\right)
\end{gathered}
$$

Now,

$$
\frac{c_{3}}{c_{1}}=\frac{\mu(\alpha+\mu)\left(\lambda_{2}+\mu\right)(\mu+\tau)\left(1-\Re_{0}\right)}{\bar{k}^{2} \sigma\left(\alpha \lambda_{1} \mu+\alpha \lambda_{3} \mu+\lambda_{3} \mu^{2}+\lambda_{1} \mu^{2}+\alpha \lambda_{3} \tau+\lambda_{1} \mu+\lambda_{3} \mu \tau+\alpha \lambda_{1} \tau(1-h m)\right)}
$$

From above, we see that $\frac{c_{3}}{c_{1}}<0$, when $\mathfrak{R}_{0}>1$.

Therefore, when $\Re_{0}>1$, we get the unique rumor-prevailing equilibrium $\bar{E}^{*}\left(I^{*}, V^{*}, E^{*}, S^{*}, R^{*}\right)$ of the model (1). According to the above result, we claim the following:

Theorem 3.6.1: The model (1) has a unique rumor-prevailing equilibrium whenever $\mathfrak{R}_{0}>1$.

\section{Numerical Simulation and Discussion}

In this section, we carry out simulations using Runge-Kutta method to investigate the dynamics of the model (1). We choose the entering rate and the leaving rate of the population as $\beta=0.1$ and $\mu=0.1$ respectively and the initial values are $I(0)=1, V(0)=0, E(0)=0, S(0)=0.2, R(0)=0$.

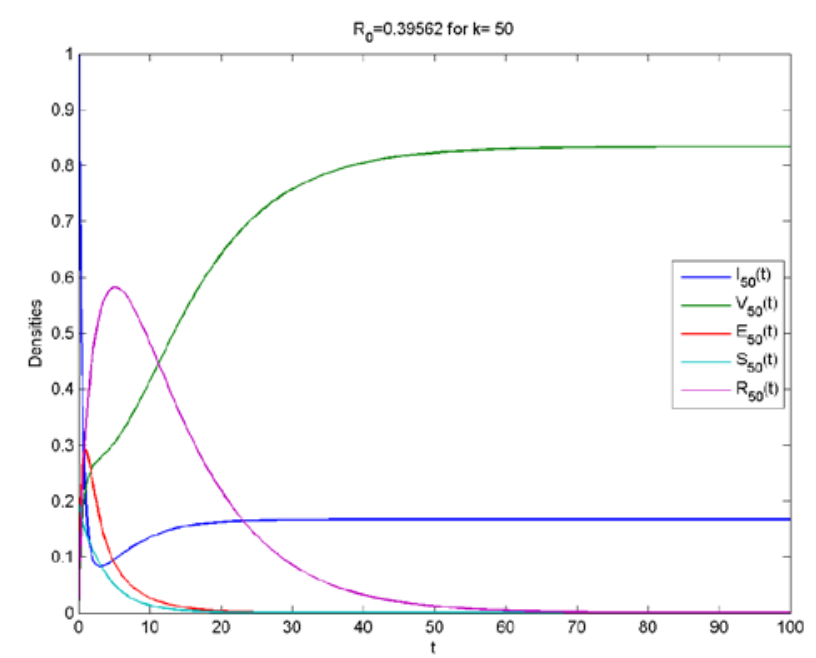

(a) $\bar{k}=50$

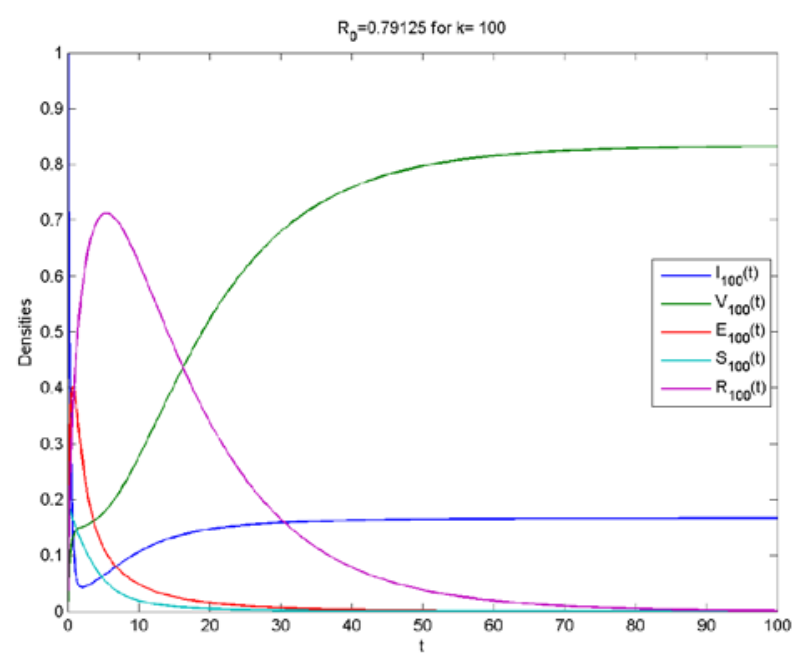

(b) $\bar{k}=100$

Figure 2. The curves of the density of the five groups over time when $\mathfrak{R}_{\mathbf{0}}<1$.

Figure 2 illustrates the change of the density of five different groups (Ignorants, Vaccinated, Exposed, Spreader, Stif- 
lers) over time with average degree $\bar{k}=50$ (Figure 2a) and $\bar{k}=100$ (Figure 2b) of the homogeneous network and the basic reproduction number is $\Re_{0}=0.39562$ for $\bar{k}=50$ and $\Re_{0}=0.79125$ for $\bar{k}=100$. We see that, with the change of time, no matter how many people spread rumor, rumor will disappear and there exist only the ignorants and vaccinated in the whole system. It suggests that the rumor-free equilibrium is globally asymptotically stable when $\mathfrak{R}_{0}<1$. Moreover, it is observed that the smaller the degree, the quicker the time to achieve maximum value for the ignorants and vaccinated and also the stiflers tend to zero quickly for smaller $\bar{k}$.

Figure 3 illustrates the change of the density of five different groups over time with average degree $\bar{k}=50$ (Figure 3a) and $\bar{k}=100$ (Figure $3 \mathrm{~b}$ ) of the homogeneous network. In this case, the basic reproduction number is $\mathfrak{R}_{0}=1.7133$ for $\bar{k}=50$ and $\mathfrak{R}_{0}=3.4267$ for $\bar{k}=100$. Figure 3 depicts that when $\mathfrak{R}_{0}>1$, even for a small fraction of the spreader nodes at the beginning the rumor is permanent in the network that is the rumor will tend to a positive stable state and when $\bar{k}=50$, then the density of the ignorants and vaccinated are larger than $\bar{k}=100$ in a stable state. But when $\bar{k}=100$, then the density of the stiflers is larger than $\bar{k}=50$ in a stable state.

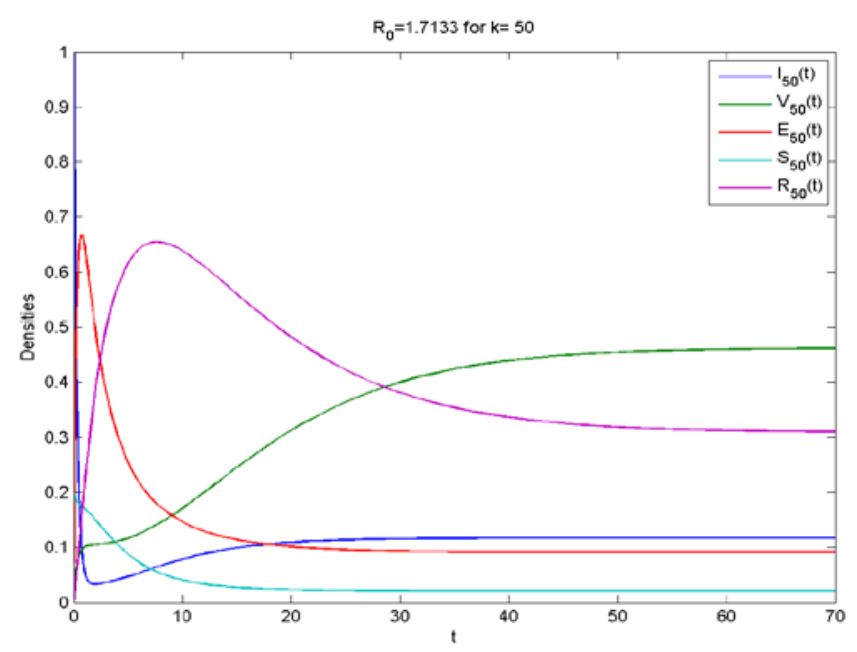

(a) $\overline{\mathrm{k}}=50$

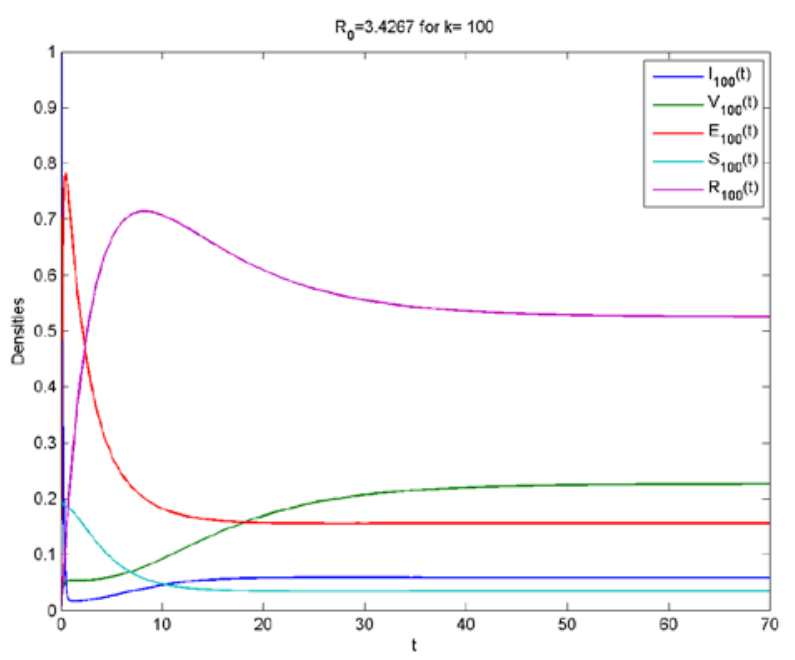

(b) $\overline{\mathrm{k}}=100$

Figure 3. The curves of the density of the five groups over time when $\mathfrak{R}_{0}>1$.

Figure 4 shows the dynamical behavior of $S_{k}$ while $\mathfrak{R}_{0}<1$ in (a) and $\Re_{0}>1$ in (b) with different degree. We find that the larger degree leads to a larger value of the spreading level.

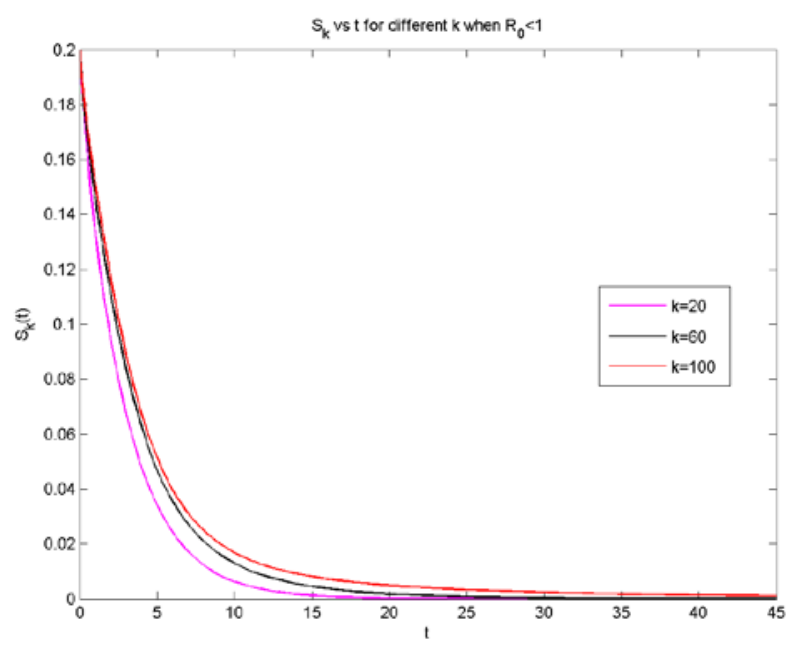

(a)

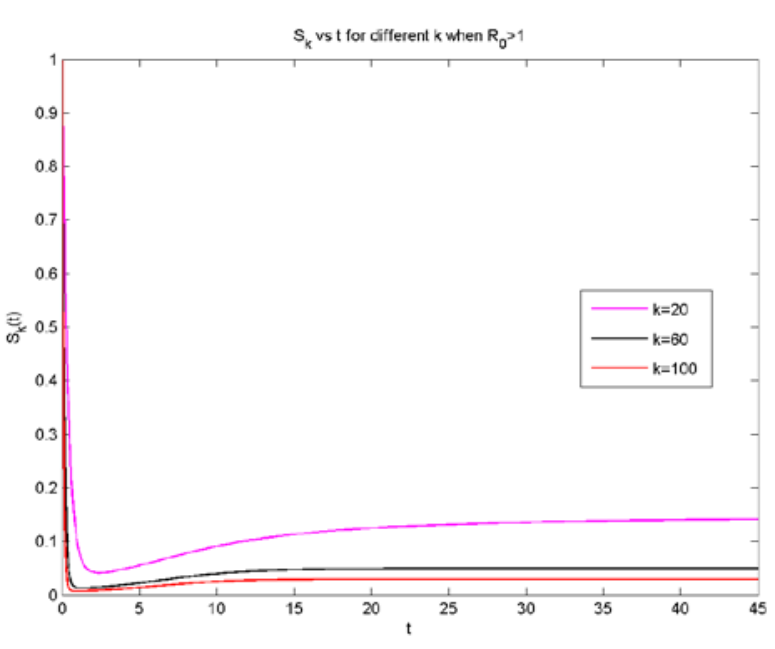

(b)

Figure 4. Dynamical behavior of $S_{k}$ in rumor-free equilibrium (a) and rumor-prevailing equilibrium (b) with different degree of network $\overline{\mathbf{k}}$. 
In Figure 5, the effect of fuzzy parameter $m$ to each population is presented. In this figure, we choose $m=0.1$ in (a) and $m=0.8$ in (b). Then we see that if we increase the value of the fuzzy parameter $m$ then the level of stiflers will decrease and the level of spreaders and exposed will increase. In reality, if the rumor is fuzzier then the people will be more curious about the rumor. So, in order to reduce the final rumor size, the respective organizations or media should give precise and clear information.

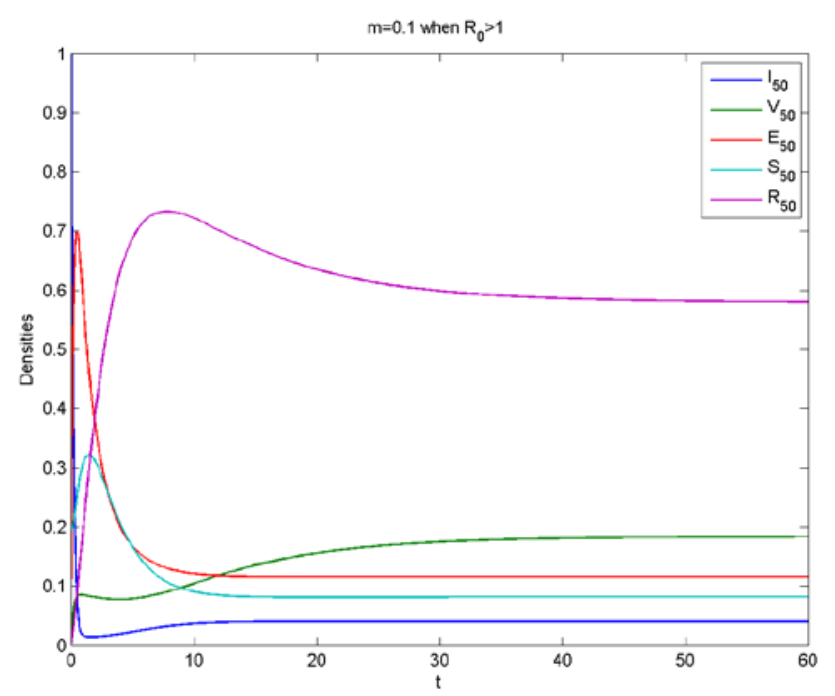

(a)

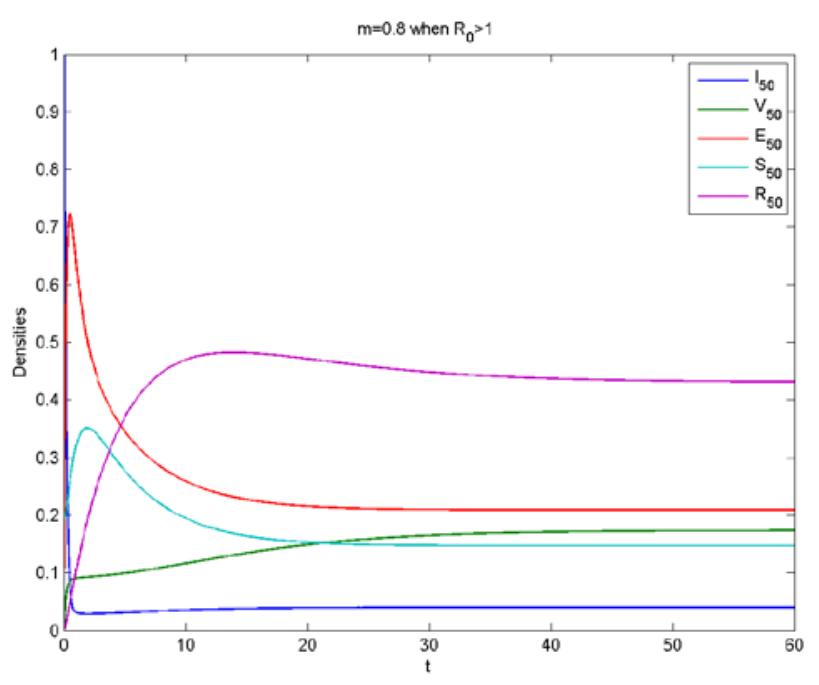

(b)

Figure 5. The curves of the density of the five groups over time with different fuzzy parameter $\mathbf{m}$.

Figure 6 shows the effect of vaccination rate parameter $\lambda_{2}$ to each population. In Figure 6, we choose $\lambda_{2}=0.2$ in (a) and $\lambda_{2}=0.6$ in (b). Then we see that if we increase the vaccination rate $\lambda_{2}$ then it will decrease the level of spreaders, exposed and stiflers. In the real world, if we increase the restriction to the spread of the rumor strictly then it will help to reduce the final size of the infected individuals.

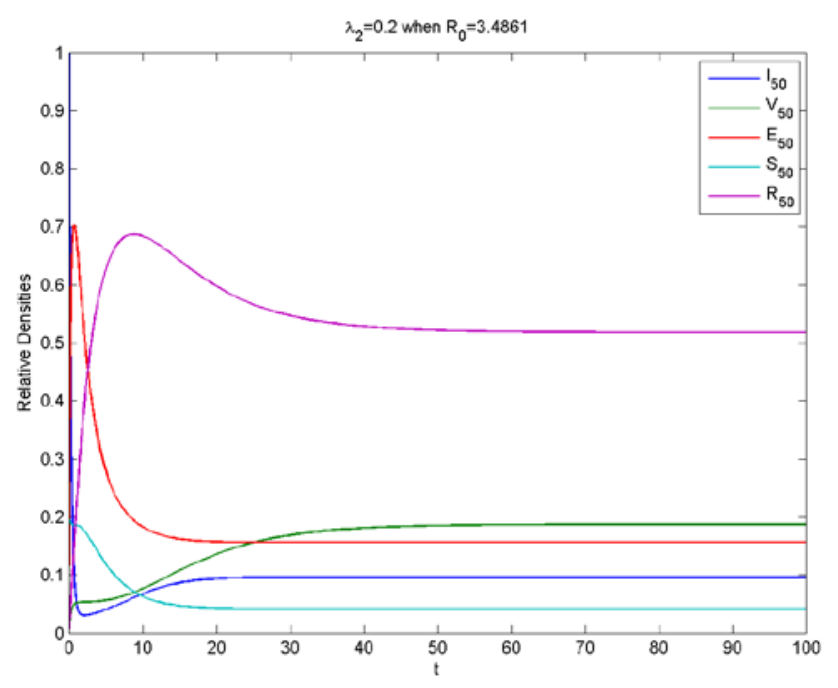

(a)

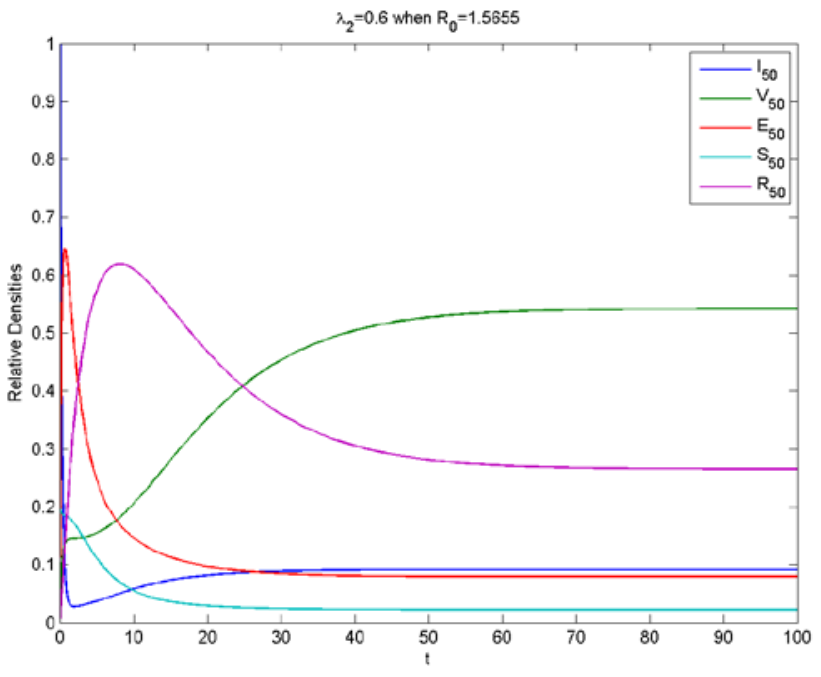

(b)

Figure 6. The curves of the density of the five groups over time with different vaccination rate $\lambda_{2}$.

Figure 7 shows the dynamical behavior of $S_{50}$ while $\Re_{0}<1$ in (a) and $\mathfrak{R}_{0}>1$ in (b) with different $\lambda_{2}$. We find that the larger value of $\lambda_{2}$ leads to a smaller value of the spreading level.

In Figure 8, the dynamical behavior of $S_{50}$ is shown when $\Re_{0}<1$ in (a) and $\Re_{0}>1$ in (b) with different $\sigma$. We find that the larger value of $\sigma$ leads to a larger value of the spreading level. 


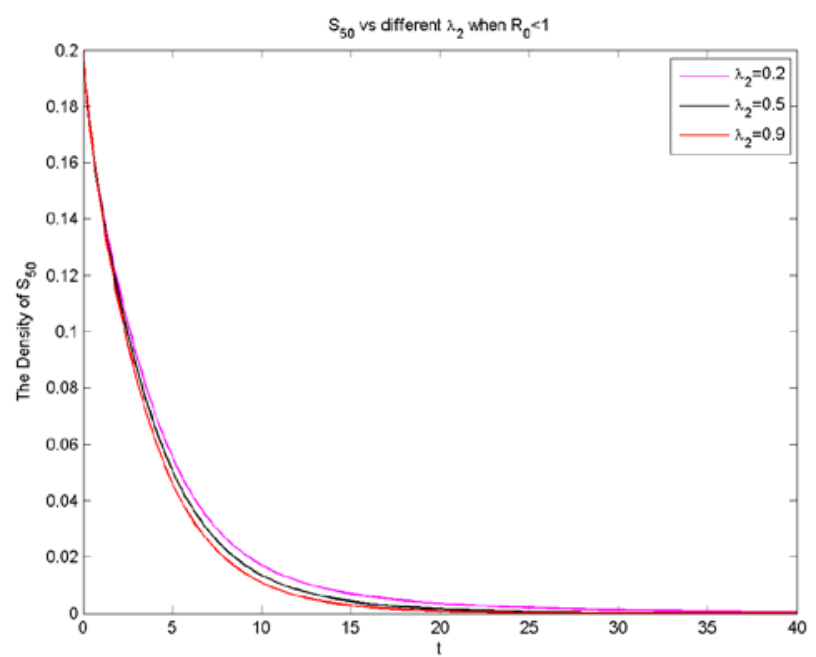

(a)

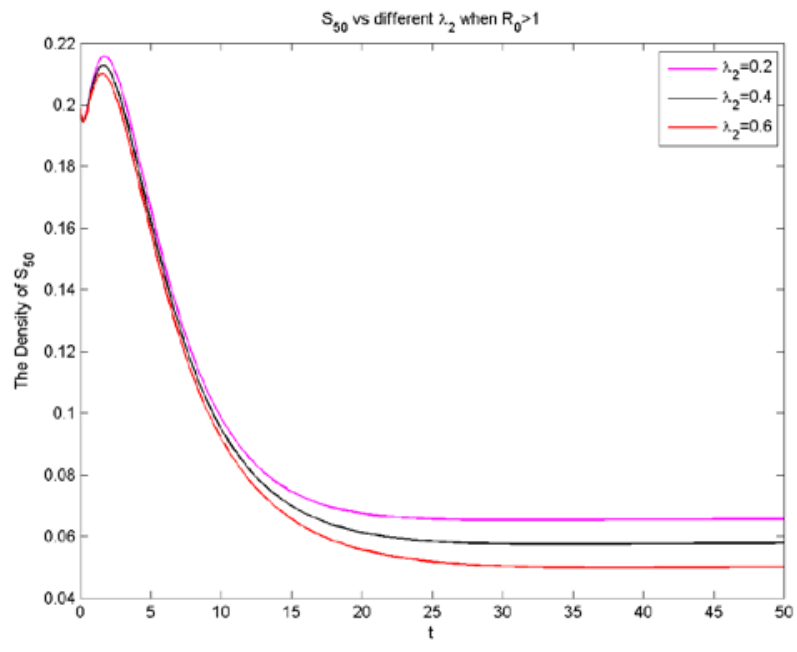

(b)

Figure 7. Dynamic behavior of $S_{50}$ in rumor-free equilibrium (a) and rumor-prevailing equilibrium (b) with different rate of vaccination $\lambda_{2}$.

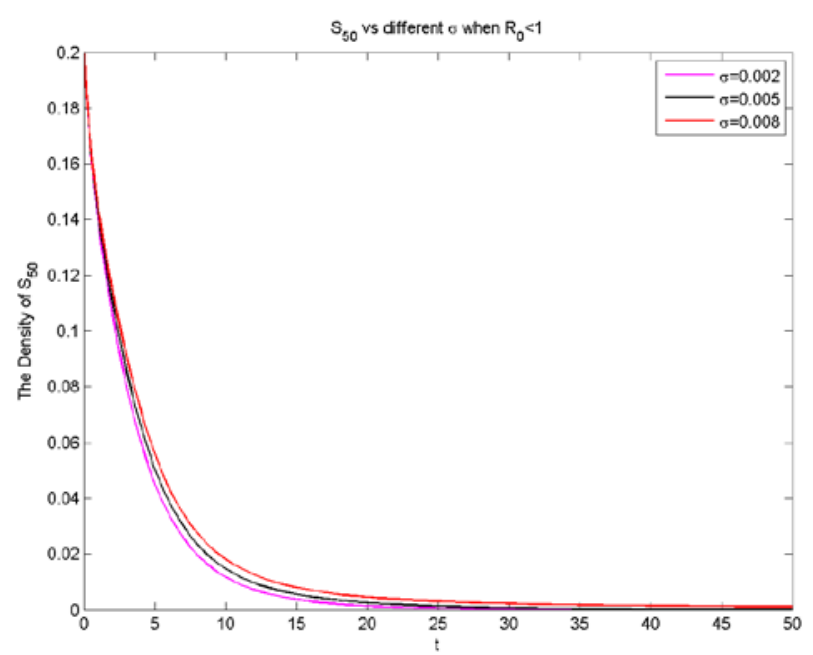

(a)

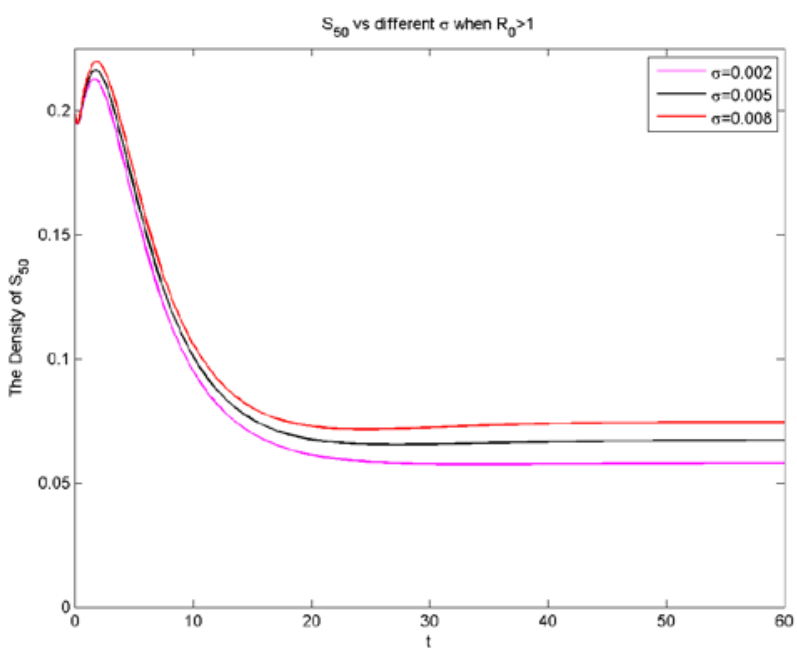

(b)

Figure 8. Dynamic behavior of $S_{50}$ in rumor-free equilibrium (a) and rumor-prevailing equilibrium (b) with different value of $\sigma$.

Figure 9 shows the effect of forgetting rate on the five compartments. In Figure 9, we choose $\alpha=0.2$ in (a) and $\alpha=0.5$ in (b). We see that, higher value of $\alpha$ results in less time for every population to reach the stable rumor-free equilibrium. That is, the more people forget the rumor the more quickly the rumor will diminish from society.

\section{Sensitivity Analysis}

We calculate the sensitivity indices of the basic reproduction number $\mathfrak{R}_{0}$ to the parameters in the model (1) to determine which of the parameter have high impact on $\mathfrak{R}_{0}$, and consequently to the rumor transmission. We follow the approach as in [27]. The normalized sensitivity indices of $\mathfrak{R}_{0}$ on parameter $P_{i}$ is given by

$$
\Upsilon_{P_{i}}^{\Re_{0}}=\frac{\partial \Re_{0}}{\partial P_{i}} \frac{P_{i}}{\Re_{0}}
$$

We evaluate the sensitivity indices at the baseline parameter values given in Table 3 , and the obtained sensitivity index of $\mathfrak{R}_{0}$ with respect to the model parameters are presented in Table 2 . In general, when one of the parameters with posi- 
tive sign increases (or decreases) while the other parameters are constant, the value of $\mathfrak{R}_{0}$ increases (or decreases) and when one of the parameters with negative sign increases (or decreases) while the other parameters are constant, the value of $\Re_{0}$ decreases (or increases).

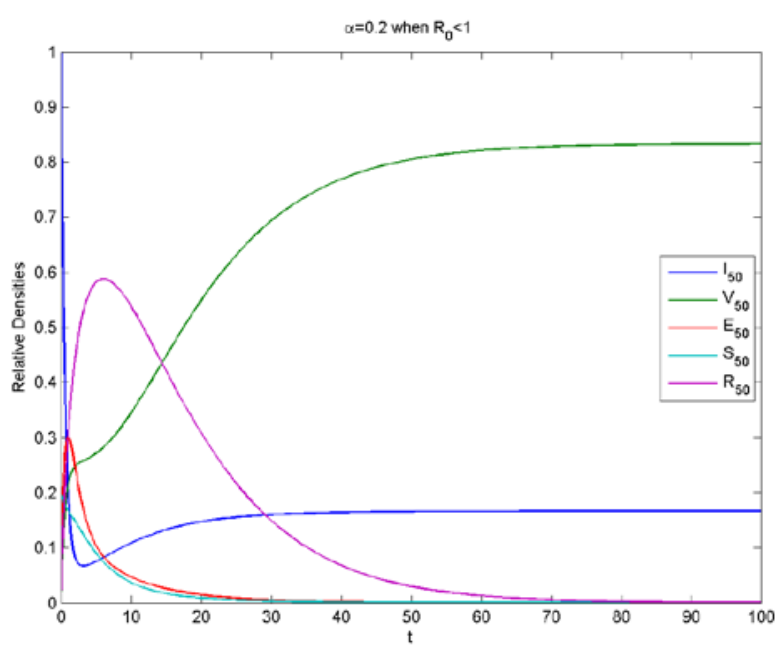

(a)

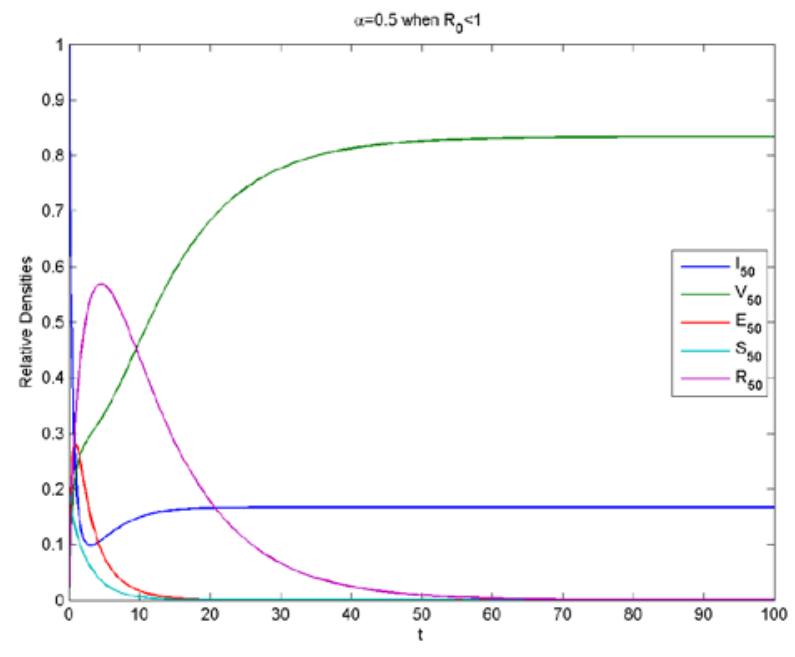

(b)

Figure 9. The curves of the density of the five groups over time with different forgetting rate $\alpha$.

Table 2. Sensitivity indices of $\mathfrak{R}_{0}$ to parameters for the model (1), evaluated at the parameter values given in Table 3

\begin{tabular}{cc}
\hline Parameters & Sensitivity Indices \\
\hline $\boldsymbol{\beta}$ & 1.00 \\
$\lambda_{1}$ & -0.762196 \\
$\lambda_{2}$ & 0.765957 \\
$\sigma$ & -0.599291 \\
$\tau$ & 0.234043 \\
$h$ & 0.139265 \\
$\alpha$ & 0.765957 \\
$\bar{k}$ & -0.777778 \\
\hline
\end{tabular}

Table 3. Values of the parameters of the model (1) for simulation

\begin{tabular}{ccc}
\hline Parameter & Value & Reference \\
\hline $\boldsymbol{\beta}$ & 0.1 & {$[20]$} \\
$\boldsymbol{\mu}$ & 0.1 & {$[20]$} \\
$\boldsymbol{\lambda}_{\mathbf{1}}$ & 0.1 & estimated \\
$\boldsymbol{\lambda}_{\mathbf{2}}$ & 0.5 & estimated \\
$\boldsymbol{\sigma}$ & 0.001 & estimated \\
$\boldsymbol{h}$ & 0.2 & [20] \\
$\boldsymbol{\tau}$ & 0.45 & estimated \\
$\boldsymbol{\alpha}$ & 0.35 & [20] \\
$\boldsymbol{k}$ & 100 & estimated
\end{tabular}

Table 2 shows that all the parameters have either positive or negative effects on the reproduction number $\mathfrak{R}_{0}$. From the table, it is clear that increasing (decreasing) $h$ by $10 \%$ will increase (decrease) $\Re_{0}$ by $0.765957 \times 10 \%$ and 
increasing (decreasing) $\mu$ by $10 \%$ will decreases (increases) $\mathfrak{R}_{0}$ by $10 \% \times|-0.762196|$. The most crucial parameters are $\beta, \bar{k}, \mu, \lambda_{1}, h$, and $\alpha$. Other important parameters are $\lambda_{2}, \sigma$, and $\tau$. The results have been shown in Figure 10 and Figure 11 which depict how $\mathfrak{R}_{0}$ is changing with different parameters at the baseline values. We observe that the entering rate of ignorants $\beta$ and the average degree of network $\bar{k}$ has the most positive effect on $\mathfrak{R}_{0}$ and the leaving rate $\mu$, vaccination rate $\lambda_{2}$ and forgetting rate $\alpha$ have negative effect on $\mathfrak{R}_{0}$. It is noticeable that, $\mathfrak{R}_{0}$ is an increasing function of the parameter with the positive valued indices and decreasing function with negative valued indices.

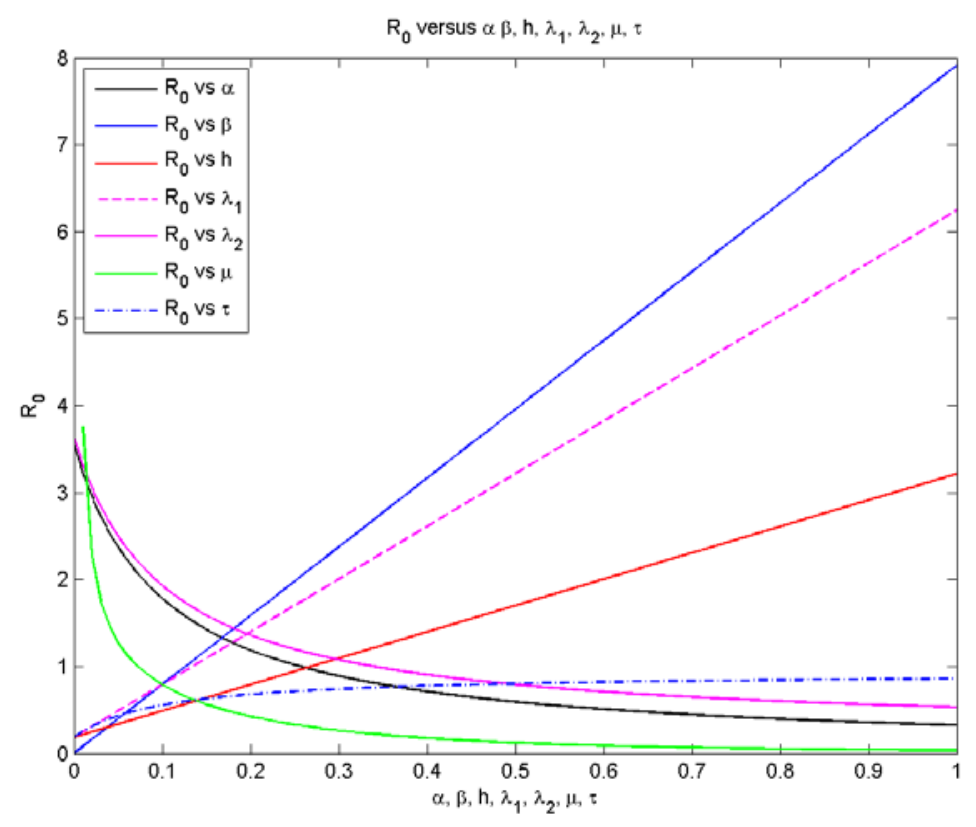

Figure 10. Sensitivity of $\mathfrak{R}_{0}$ with respect to the parameters $\alpha, \beta, h, \lambda_{1}, \lambda_{2}, \mu$ and $\tau$.

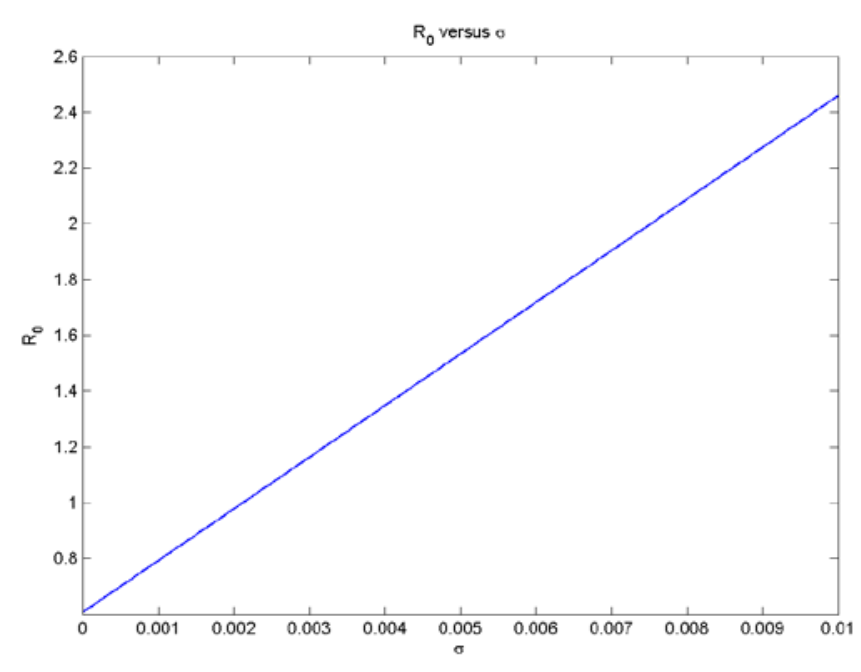

(a)

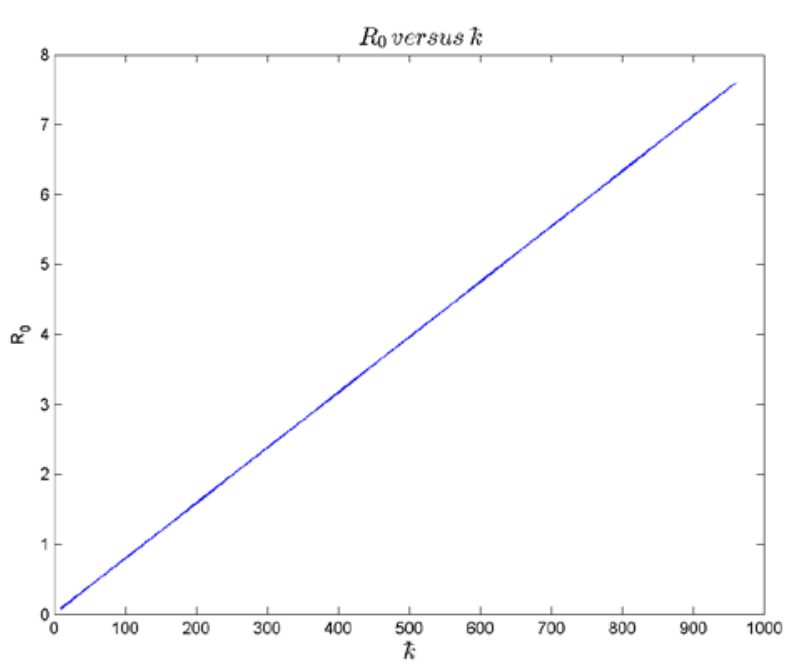

(b)

Figure 11. Sensitivity of $\mathfrak{R}_{0}$ with respect to (a) parameter $\sigma$ and (b) average degree of the net work $\bar{k}$.

\section{Conclusion}

In this article, a new IVESR rumor spreading model with hesitating and forgetting mechanism has been formulated and analyzed rigorously. We have shown that the model has a rumor free equilibrium which is locally as well as globally asymptotically stable when the threshold parameter $\mathfrak{R}_{0}$ is less than unity. That is, if the quantity $\mathfrak{R}_{0}$ is less than one, the rumor will not persist in the network. Also, the existence of the rumor prevailing equilibrium has been proved and it 
exists whenever $\mathfrak{R}_{0}>1$. Moreover, the disallowance to the rumor spreading by authoritative order or the consciousness campaign can help to reduce the speed of spreading of the rumor as the vaccination rate $\lambda_{2}$ has a great negative effect on $\mathfrak{R}_{0}$. However, the increasing transfer rate from vaccinated to spreader accelerates the spreading of the rumor. We also have found out that the fuzziness of the rumor increases the label of propagation of the rumor in the network and the higher forgetting rate reduces the prevailing duration of the rumor.

\section{References}

[1] Galam, S. (2003). Modelling rumors: the no plane Pentagon French hoax case. Physica A: Statistical Mechanics and Its Applications, 2003, 320: 571-580.

[2] Hayakawa, H. (2002). Sociology of rumor-approach from formal sociology. Seikyusya, Tokyo, 2002.

[3] Kimmel, A. J. (2004). Rumors and rumor control: A manager's guide to understanding and combatting rumors. Routledge.

[4] Kosfeld, M. (2005). Rumours and markets. Journal of Mathematical Economics, 2005, 41(6): 646-664.

[5] Kawachi, K. (2008). Deterministic models for rumor transmission. Nonlinear analysis: Real world applications, 2008, 9(5): 1989-2028.

[6] Misra, A. K. (2012). A simple mathematical model for the spread of two political parties. Nonlinear Analysis: Modelling and Control, 2012, 17(3): 343-354.

[7] Daley, D. J. and D. G. Kendall. (1964). Epidemics and rumours. Nature, 1964, 204(4963): 1118-1118.

[8] Kimmel, A. J. (2004). Rumors and the financial marketplace. The Journal of Behavioral Finance, 2004, 5(3): 134-141.

[9] Barabási, A.-L. and R. Albert. (1999). Emergence of scaling in random networks. Science, 1999, 286(5439): 509-512.

[10] Kesten, H. and V. Sidoravicius. (2005). The spread of a rumor or infection in a moving population. Annals of Probability, 2005, 33(6): 2402-2462.

[11] Daley, D. J. and D. G. Kendall. (1965). Stochastic rumours. IMA Journal of Applied Mathematics, 1965, 1(1): 42-55.

[12] Maki, D. P. and M. Thompson. (1973). Mathematical models and applications: with emphasis on the social life, and management sciences.

[13] Nekovee, M., et al. (2007). Theory of rumour spreading in complex social networks. Physica A: Statistical Mechanics and its Applications, 2007, 374(1): 457-470.

[14] Isham, V., S. Harden, and M. Nekovee. (2010). Stochastic epidemics and rumours on finite random networks. Physica A: Statistical Mechanics and its Applications, 2010, 389(3): 561-576.

[15] Gu, J. and X. Cai. (2007). The forget-remember mechanism for 2-state spreading. arXiv preprint nlin/0702021.

[16] Gu, J., W. Li, and X. Cai. (208). The effect of the forget-remember mechanism on spreading. The European Physical Journal B, 2008, 62(2): 247-255.

[17] Zhao, L., et al. (2013). Rumor spreading model considering forgetting and remembering mechanisms in inhomogeneous networks. Physica A: Statistical Mechanics and its Applications, 2013, 392(4): 987-994.

[18] Zhao, L., et al. (2012). SIHR rumor spreading model in social networks. Physica A: Statistical Mechanics and its Applications, 2012, 391(7): 2444-2453.

[19] Wan, C., T. Li, and Z. Sun. (2017). Global stability of a SEIR rumor spreading model with demographics on scale-free networks. Advances in Difference Equations, 2017(1): 1-15.

[20] Liu, X., T. Li, and M. Tian. (2018). Rumor spreading of a SEIR model in complex social networks with hesitating mechanism. Advances in Difference Equations, 2018(1): 1-24.

[21] Zhou, Y., et al. (2019). Rumor source detection in networks based on the SEIR model. IEEE access, 2019, 7: 45240-45258.

[22] Lakshmikantham, V., S. Leela, and A. A. Martynyuk. (1989). Stability analysis of nonlinear systems. 1989: Springer. 
[23] Hethcote, H. W. (2000). The mathematics of infectious diseases. SIAM review, 2000, 42(4): 599-653.

[24] Van den Driessche, P. and J. Watmough. (2002). Reproduction numbers and sub-threshold endemic equilibria for compartmental models of disease transmission. Mathematical biosciences, 2002, 180(1-2): 29-48.

[25] Hale, J. (1969). Ordinary Differential Equations Wiley. New York. 1969.

[26] La Salle, J. P. (1976). The stability of dynamical systems. 1976: SIAM.

[27] Chitnis, N., J. M. Hyman, and J. M. Cushing. (2008). Determining important parameters in the spread of malaria through the sensitivity analysis of a mathematical model. Bulletin of Mathematical Biology, 2008, 70(5): 1272. 